\title{
非线性超声检测方法的应用与挑战
}

项延训

华东理工大学机械与动力工程学院, 上海 200237

E-mail: yxxiang@ecust.edu.cn

\section{Applications and challenges of nonlinear ultrasound testing}

\section{Yanxun Xiang}

School of Mechanical and Power Engineering, East China University of Science and Technology, Shanghai 200237, China E-mail: yxxiang@ecust.edu.cn

doi: 10.1360/TB-2022-0136

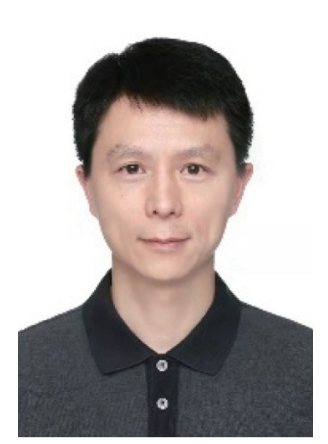

\section{项延训}

华东理工大学机械与动力工程学 院教授, 博士生导师; 中国声学学 会理事, 中国声学学会检测声学分 会委员, 中国声学学会物理声学分 会委员。主要从事机械结构服役状 态非线性超声检测、超声导波检 测及成像、检测数据挖掘与机器 学习等研究.
材料损伤的早期发现和预防是避免破坏性事故的有效手段，日渐成为重大 装备安全服役领域的研究前沿和热点. 超声波因其传播特性与材料性能直接相 关而被广泛应用于检测和评估材料损伤。传统的超声检测方法可以有效检测的 缺陷尺寸通常限制在半波长以上, 仅能适用于材料损伤中后期的宏观缺陷(如宏 观裂纹、孔洞和夹杂物等)的检测，对材料损伤早期的性能退化不太敏感. 近年 研究表明 ${ }^{[1,2]}$, 材料性能退化、演化或微裂纹等微弱变化会引起介质中超声波传 播的非线性效应. 由此发展起来的非线性超声理论与技术广受研究者们关注, 研 究通常涉及与材料弹性非线性相关的非线性超声检测及与裂纹接触声非线性相 关的非线性超声检测. 这种检测方法可以克服传统超声检测方法对材料微弱性 能变化不敏感的局限性，有效地评估材料的性能退化程度或微裂纹的大小、状 态等参数, 为损伤早期发现和预防提供了新手段.

为了报道非线性超声原理及检测方法的最新研究进展, 《科学通报》邀请 国内部分专家学者, 组织了“非线性超声检测方法”专题, 内容涉及非线性超声 发生效应理论 ${ }^{[3,4]}$ 及其在材料退化 ${ }^{[3]}$ 、泰接状态 ${ }^{[5,6]}$ 和微裂纹 ${ }^{[4]}$ 检测方面的研究进 展. 专题阐述了非线性超声检测方法在基础理论、数值模拟和实验测量方面的 研究现状、前沿进展和未来展望, 方法涉及高次谐波、次谐波、直流分量、混 频和非线性谐振等, 希望可以帮助读者们系统了解这一领域的前沿发展.

\section{参考文献}

1 Xiang Y X, Deng M X, Xuan F Z. Creep damage characterization using nonlinear ultrasonic guided wave method: A mesoscale model. J Appl Phys, 2014, 115: 044914

2 Sun M X, Xiang Y X, Deng M X, et al. Experimental and numerical investigations of nonlinear interaction of counter-propagating Lamb waves. Appl Phys Lett, 2019, 114: 011902

3 Li W B, Xiang Y X, Deng M X. Advances in theoretical, experimental and applied studies on second-harmonic generation of ultrasonic Lamb waves (in Chinese). Chin Sci Bull, 2022, 67: 583-596 [李卫彬, 项延训, 邓明晰. 超声兰姆波二次谐波发生效应的理论、实验及应用研究进展. 科学通报, 2022, 67: 583-596] 
4 Sun D, Zhu W J, Xiang Y X, et al. Advances in nonlinear ultrasonic detection of microcracks (in Chinese). Chin Sci Bull, 2022, 67: 597-609 [孙迪, 朱武军, 项延训, 等. 微裂纹的非线性超声检测研究进展. 科学通报, 2022, 67: 597-609]

5 Li X Y, Shui G S, Wang Y S, et al. Progress on nonlinear ultrasonic evaluation of damages within the adhesively bonded composites (in Chinese). Chin Sci Bull, 2022, 67: 610-620 [李晓阳, 税国双, 汪越胜, 等. 秥接复合结构损伤的非线性超声评价研究进展. 科学通报, 2022, 67: 610-620]

6 Li L, Jiao J P, Gao X, et al. A review on nondestructive testing of bonding interface using nonlinear ultrasonic technique (in Chinese). Chin Sci Bull, 2022, 67: 621-629 [李立, 焦敬品, 高翔, 等. 黏接界面性能劣化的非线性超声检测研究进展. 科学通报, 2022, 67: 621-629] 